Supplemental material for

\title{
Fluorimetry compared to spectrophotometry for Uranium evaluation in liquid waste
}

Aurelia Magdalena Dianu $^{\mathrm{a}^{*}}$ and Marius Iordache ${ }^{\mathrm{a}}$

${ }^{a}$ Radioactive Waste Treatment Plant, Institute for Nuclear Research, Pitesti, Romania

Table S1. Uncertainty budgets for the spectrophotometer and the calibration standards

Table S2. Uncertainty of the spectrophotometric method, expressed in $\left[\mathrm{mg} \mathrm{L}^{-1}\right]$

Table S3. Raw data for the uranium concentration measurements by the two methods, expressed in [mg L $\mathrm{m}^{-1}$ ]

Table S4. Grubbs test for the fluorimetric method

Figure S1. Calibration curve for the spectrophotometric method

Figure S2. Cause and effect diagram for uncertainty sources of the spectrophotometric method

Figure S3. Relation between uncertainty and concentration for the spectrophotometric method

${ }^{*}$ Corresponding author. Email: mdianu@yahoo.com 
To compute the standard uncertainties of the spectrophotometer $u(S P)$ and the calibration standards $u(C S)$, the uncertainty budgets were constructed based on the technical specification provided by the apparatus producer and the passport certificate of the standard reference materials. These budgets are presented in (Table S1).

Table S1. Uncertainty budgets for the spectrophotometer and the calibration standards

\begin{tabular}{|c|c|c|c|c|c|c|c|}
\hline ID & Parameter & $\begin{array}{l}\text { Limits of } \\
\text { variation } \pm v\end{array}$ & $\begin{array}{l}\text { Distribution } \\
\text { type }\end{array}$ & $\begin{array}{l}\text { Evaluation } \\
\text { method [5] }\end{array}$ & Range, $2 v$ & $\begin{array}{l}\text { Standard } \\
\text { uncertainty, } \\
u(X)\end{array}$ & $\begin{array}{l}\text { Relative standard } \\
\text { uncertainty, } \\
u r(X)=\mathrm{u}(X) / X\end{array}$ \\
\hline$A b s 1$ & $\begin{array}{l}\text { Photometric } \\
\text { reproductibility }\end{array}$ & $\pm 0.005^{*} A b s$ & Rectangular & $(0.5 * 2 v / \sqrt{3})$ & $0.01 * A b s$ & $0.00288675^{*} A b s$ & 0.00288675 \\
\hline$A b s 2$ & $\begin{array}{l}\text { Photometric } \\
\text { repeatability }\end{array}$ & $\pm 0.001 * A b s$ & Rectangular & $(0.5 * 2 v / \sqrt{3})$ & $0.002 * A b s$ & $0.00057735 * A b s$ & 0.00057735 \\
\hline$A b s 3$ & Photometric linearity & $\pm 0.002 * A b s$ & Rectangular & $(0.5 * 2 v / \sqrt{3})$ & $0.004^{*} A b s$ & $0.0011547 * A b s$ & 0.0011547 \\
\hline$A b s 4$ & Noise level & $\begin{array}{l} \pm \\
0.0001 * A b s\end{array}$ & Rectangular & $(0.5 * 2 v / \sqrt{3})$ & $0.0002 * A b s$ & $5.7735 \mathrm{E}-05^{*} A b s$ & $5.7735 \mathrm{E}-05$ \\
\hline$A b s 5$ & $\begin{array}{l}\text { Drift } \\
\left(25^{\circ} \mathrm{C} ; 15 \text { minutes }\right)\end{array}$ & $\pm 0.001 * A b s$ & Rectangular & $(0.5 * 2 v / \sqrt{3})$ & $0.002 * A b s$ & $0.00057735 * A b s$ & 0.00057735 \\
\hline$A b s$ & $\begin{array}{l}\text { Photometric } \\
\text { uncertainty }\end{array}$ & $u(A b s)=\sqrt{u(A b s}$ & $1)^{2}+u(A b s 2)^{2}+u$ & $A b s 3)^{2}+u(A b s 4$ & )$^{2}+u(A b s 5)^{2}$ & $0.00321507 * A b s$ & 0.00321507 \\
\hline$D$ & Display uncertainty & 4 digits & Rectangular & $(0.5 * 2 v / \sqrt{3})$ & 0.001 & 0.00028868 & \\
\hline$W 1$ & $\begin{array}{l}\text { Wavelength accuracy } \\
(X \text { set to } 665 \mathrm{~nm})\end{array}$ & $\pm 1 \mathrm{~nm}$ & Triangular & $(0.5 * 2 v / \sqrt{6})$ & 2 & 0.40824829 & 0.000613907 \\
\hline$W 2$ & $\begin{array}{l}\text { Wavelength } \\
\text { repeatability } \\
(X \text { set to } 665 \mathrm{~nm})\end{array}$ & $\pm 0.2 \mathrm{~nm}$ & Rectangular & $(0.5 * 2 v / \sqrt{3})$ & 0.4 & 0.11547005 & 0.000173639 \\
\hline$W 3$ & $\begin{array}{l}\text { Wavelength } \\
\text { resolution } \\
(X \text { set to } 665 \mathrm{~nm})\end{array}$ & $0.1 \mathrm{~nm}$ & Rectangular & $(0.5 * 2 v / \sqrt{3})$ & 0.1 & 0.02886751 & $4.34098 \mathrm{E}-05$ \\
\hline$W$ & $\begin{array}{l}\text { Wavelength } \\
\text { relative uncertainty }\end{array}$ & \multicolumn{5}{|c|}{$u r(W)=\sqrt{u r(W 1)^{2}+u r(W 2)^{2}+u r(W 3)^{2}}$} & 0.00063947 \\
\hline
\end{tabular}




\begin{tabular}{|c|c|c|c|c|c|c|}
\hline$S L$ & Stray light & $\begin{array}{l}<0.1 \% \text { from } \\
\text { transmittance Rectangular } \\
(1 / A b s)\end{array}$ & $(0.5 * 2 v / \sqrt{3})$ & 0.001 & $0.00028868 / A b s$ & 0.00028868 \\
\hline$M$ & $\begin{array}{l}\text { Monochromator } \\
\text { relative uncertainty }\end{array}$ & $u r(M)=\sqrt{u r(W)^{2}+u r(S L)^{2}}$ & & & & 0.00070161 \\
\hline$S P$ & $\begin{array}{l}\text { Spectrophotometer } \\
\text { uncertainty }\end{array}$ & $u(S P)=\sqrt{u(A b s)^{2}+u(D)^{2}+u(M)^{2}}$ & & & 0.00335229 & \\
\hline$C S$ & $\begin{array}{l}\text { Calibration standards } \\
\left(X \text { set to } 300 \mathrm{mg} \mathrm{L}^{-1}\right)\end{array}$ & Normal (0.95) & $0.5 * 2 v * X$ & 0.01 & 1.5 & 0.005 \\
\hline
\end{tabular}


In (Table S2) are presented the calculated values for uncertainty of the spectrophotometric method, $\boldsymbol{u}_{\mathrm{REF}}$, for each of the concentration standards values. It is observed that the greatest influence in $\boldsymbol{u}_{\boldsymbol{R} E \boldsymbol{F}}$ is due to uncertainty of the calibration curve, $\boldsymbol{u}[\boldsymbol{C}(\boldsymbol{x})]$.

Table S2. Uncertainty of the spectrophotometric method, expressed in $\left[\mathrm{mg} \mathrm{L}^{-1}\right]$

\begin{tabular}{ll|llllll|l}
\hline $\boldsymbol{C}$ & $\boldsymbol{A} \boldsymbol{b} \boldsymbol{s}$ & $\boldsymbol{u}[\boldsymbol{C}(\boldsymbol{x})]$ & $\boldsymbol{u}(\boldsymbol{A b s})$ & $\boldsymbol{u}(\boldsymbol{S} \boldsymbol{L})$ & $\boldsymbol{u}(\boldsymbol{D})$ & $\boldsymbol{u}(\boldsymbol{M})$ & $\boldsymbol{u}(\boldsymbol{C S})$ & $\boldsymbol{u}_{\boldsymbol{R E F}}$ \\
\hline 0.25 & 0.113 & 0.049655052 & 0.000363303 & 0.002554647 & 0.000288675 & 0.002633465 & 0.00125 & 0.049808266 \\
0.50 & 0.241 & 0.046874186 & 0.000774832 & 0.001197822 & 0.000288675 & 0.001357827 & 0.00250 & 0.046982991 \\
1.00 & 0.532 & 0.044255852 & 0.001710417 & 0.000542622 & 0.000288675 & 0.000838663 & 0.00500 & 0.044582363 \\
1.50 & 0.762 & 0.046017964 & 0.002449882 & 0.000378839 & 0.000288675 & 0.000743260 & 0.00750 & 0.046697797 \\
2.00 & 0.997 & 0.051714668 & 0.003205423 & 0.000289544 & 0.000288675 & 0.000701963 & 0.01000 & 0.052776335 \\
\hline
\end{tabular}


The raw data, for the 18 process samples, measured by both methods, $\boldsymbol{R}$ (spectrophotometry) and $\boldsymbol{A}$ (fluorimetry), are displayed in (Table S3).

Table S3. Raw data for the uranium concentration measurements by the two methods, expressed in $\left[\mathrm{mg} \mathrm{L}^{-1}\right.$ ]

\begin{tabular}{|c|c|c|c|c|c|c|c|c|c|c|c|}
\hline Level & $R$ & $A$ & & & & & & & & & \\
\hline 1 & 0.770 & 0.766 & 0.765 & 0.767 & 0.769 & 0.767 & 0.775 & 0.775 & 0.773 & 0.776 & 0.771 \\
\hline 2 & 0.580 & 0.550 & 0.552 & 0.559 & 0.600 & 0.557 & 0.559 & 0.558 & 0.559 & 0.600 & 0.600 \\
\hline 3 & 0.090 & 0.080 & 0.082 & 0.086 & 0.088 & 0.090 & 0.091 & 0.090 & 0.089 & 0.088 & 0.086 \\
\hline 4 & 0.308 & 0.294 & 0.296 & 0.300 & 0.299 & 0.300 & 0.300 & 0.302 & 0.302 & 0.302 & 0.303 \\
\hline 5 & 0.136 & 0.130 & 0.132 & 0.135 & 0.138 & 0.139 & 0.138 & 0.140 & 0.139 & 0.140 & 0.140 \\
\hline 6 & 0.372 & 0.369 & 0.370 & 0.369 & 0.370 & 0.371 & 0.372 & 0.373 & 0.374 & 0.374 & 0.375 \\
\hline 7 & 0.250 & 0.245 & 0.246 & 0.245 & 0.248 & 0.249 & 0.250 & 0.251 & 0.252 & 0.252 & 0.252 \\
\hline 8 & 0.264 & 0.260 & 0.260 & 0.261 & 0.259 & 0.265 & 0.266 & 0.264 & 0.265 & 0.266 & 0.264 \\
\hline 9 & 0.060 & 0.050 & 0.055 & 0.055 & 0.059 & 0.060 & 0.065 & 0.062 & 0.060 & 0.059 & 0.062 \\
\hline 10 & 0.140 & 0.130 & 0.136 & 0.137 & 0.140 & 0.142 & 0.143 & 0.145 & 0.147 & 0.145 & 0.146 \\
\hline 11 & 0.114 & 0.113 & 0.112 & 0.113 & 0.115 & 0.114 & 0.116 & 0.115 & 0.117 & 0.115 & 0.115 \\
\hline 12 & 0.980 & 0.990 & 0.992 & 0.993 & 0.994 & 0.994 & 0.995 & 0.996 & 0.996 & 0.997 & 0.998 \\
\hline 13 & 0.542 & 0.535 & 0.538 & 0.540 & 0.540 & 0.543 & 0.544 & 0.545 & 0.548 & 0.549 & 0.549 \\
\hline 14 & 0.436 & 0.429 & 0.430 & 0.431 & 0.431 & 0.430 & 0.431 & 0.431 & 0.432 & 0.432 & 0.432 \\
\hline 15 & 0.894 & 0.895 & 0.896 & 0.899 & 0.900 & 0.901 & 0.900 & 0.902 & 0.901 & 0.902 & 0.901 \\
\hline 16 & 0.640 & 0.646 & 0.650 & 0.651 & 0.652 & 0.654 & 0.660 & 0.658 & 0.662 & 0.661 & 0.662 \\
\hline 17 & 0.900 & 0.916 & 0.912 & 0.918 & 0.919 & 0.921 & 0.927 & 0.929 & 0.929 & 0.931 & 0.932 \\
\hline 18 & 0.614 & 0.615 & 0.616 & 0.619 & 0.618 & 0.620 & 0.622 & 0.621 & 0.620 & 0.622 & 0.621 \\
\hline
\end{tabular}


Each measurement value of raw data for the fluorimetric method was scrutinized for one outlying observation, applying the Grubbs test and comparing the test value with the critical value of 2.29 (10 measurements for a $5 \%$ significance level) for each level. The results of the Grubbs test are shown in (Table S4).

Table S4. Grubbs test for the fluorimetric method

\begin{tabular}{|c|c|c|c|c|c|c|c|c|c|c|c|c|c|c|c|c|c|}
\hline 1 & 2 & 3 & 4 & 5 & 6 & 7 & 8 & 9 & 10 & 11 & 12 & 13 & 14 & 15 & 16 & 17 & 18 \\
\hline 1.06 & 0.91 & 1.95 & 2.03 & 1.99 & 1.22 & 1.39 & 1.11 & 2.01 & 2.06 & 0.99 & 1.86 & 1.68 & 1.91 & 1.95 & 1.67 & 1.05 & 1.82 \\
\hline 1.30 & 0.82 & 1.39 & 1.33 & 1.43 & 0.77 & 1.05 & 1.11 & 0.86 & 0.95 & 1.66 & 1.04 & 1.06 & 0.91 & 1.54 & 0.98 & 1.62 & 1.41 \\
\hline 0.82 & 0.49 & 0.28 & 0.07 & 0.59 & 1.22 & 1.39 & 0.74 & 0.86 & 0.76 & 0.99 & 0.62 & 0.64 & 0.10 & 0.29 & 0.80 & 0.77 & 0.17 \\
\hline 0.34 & 1.43 & 0.28 & 0.28 & 0.25 & 0.77 & 0.35 & 1.48 & 0.07 & 0.20 & 0.33 & 0.21 & 0.64 & 0.10 & 0.12 & 0.63 & 0.62 & 0.58 \\
\hline 0.82 & 0.58 & 0.84 & 0.07 & 0.53 & 0.32 & 0.00 & 0.74 & 0.30 & 0.17 & 0.33 & 0.21 & 0.02 & 0.91 & 0.54 & 0.28 & 0.34 & 0.25 \\
\hline 1.11 & 0.49 & 1.11 & 0.07 & 0.25 & 0.14 & 0.35 & 1.11 & 1.46 & 0.35 & 0.99 & 0.21 & 0.19 & 0.10 & 0.12 & 0.77 & 0.51 & 1.08 \\
\hline 1.11 & 0.53 & 0.84 & 0.77 & 0.81 & 0.59 & 0.70 & 0.37 & 0.76 & 0.72 & 0.33 & 0.62 & 0.39 & 0.10 & 0.96 & 0.42 & 0.80 & 0.66 \\
\hline 0.63 & 0.49 & 0.56 & 0.77 & 0.53 & 1.04 & 1.05 & 0.74 & 0.30 & 1.10 & 1.66 & 0.62 & 1.02 & 1.11 & 0.54 & 1.12 & 0.80 & 0.25 \\
\hline 1.35 & 1.43 & 0.28 & 0.77 & 0.81 & 1.04 & 1.05 & 1.11 & 0.07 & 0.72 & 0.33 & 1.04 & 1.22 & 1.11 & 0.96 & 0.94 & 1.08 & 1.08 \\
\hline 0.14 & 1.43 & 0.28 & 1.12 & 0.81 & 1.49 & 1.05 & 0.37 & 0.76 & 0.91 & 0.33 & 1.45 & 1.22 & 1.11 & 0.54 & 1.12 & 1.22 & 0.66 \\
\hline
\end{tabular}




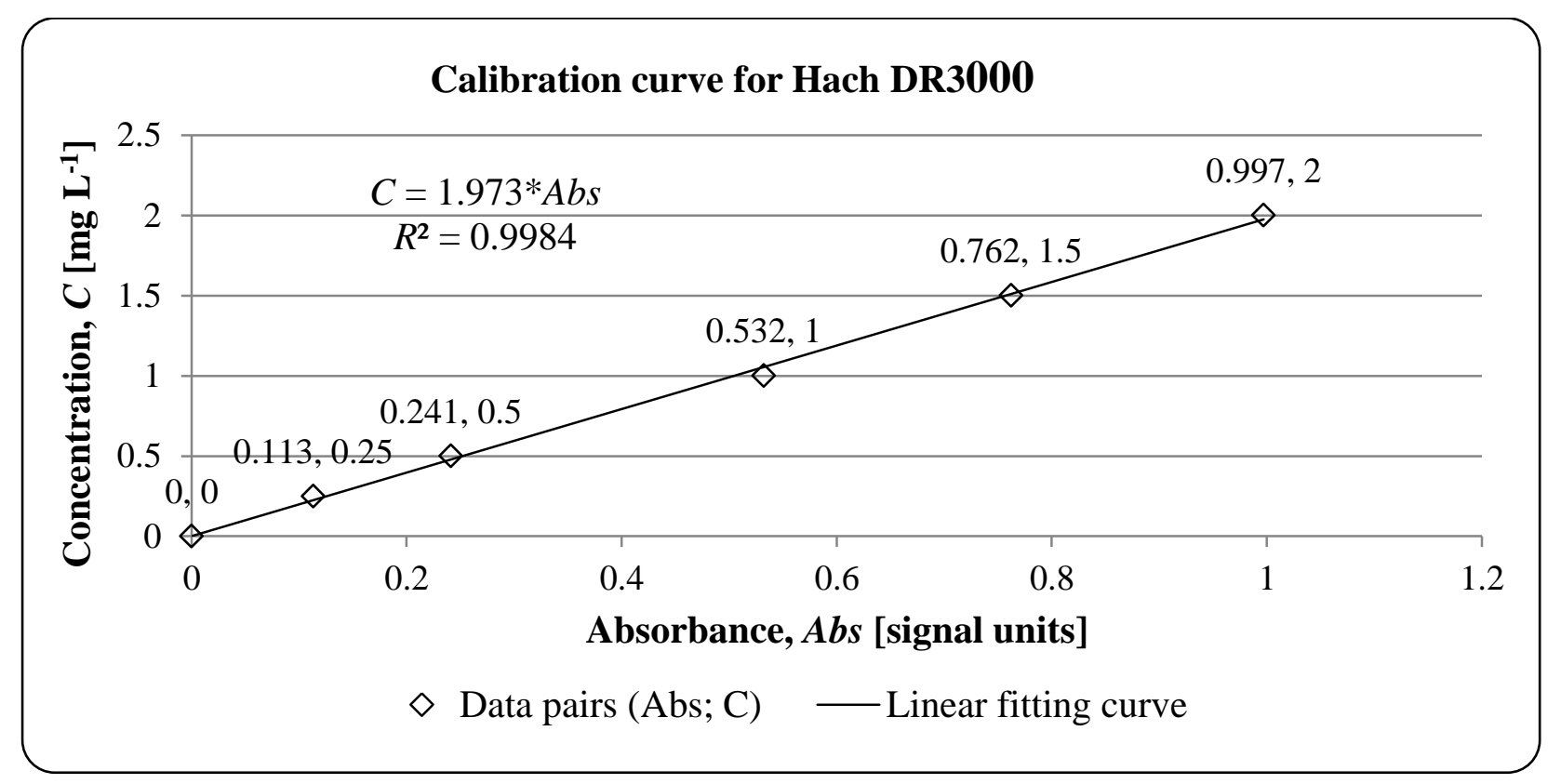

Figure S1. Calibration curve for the spectrophotometric method

In (Figure $\mathrm{S} 1$ ) is displayed the calibration curve achieved with the standard values, the curve equation and the $R$-squared value (coefficient of determination that expresses the statistical level of agreement between the approximated regression line and the real data, where value of 1 means a perfect fit). 


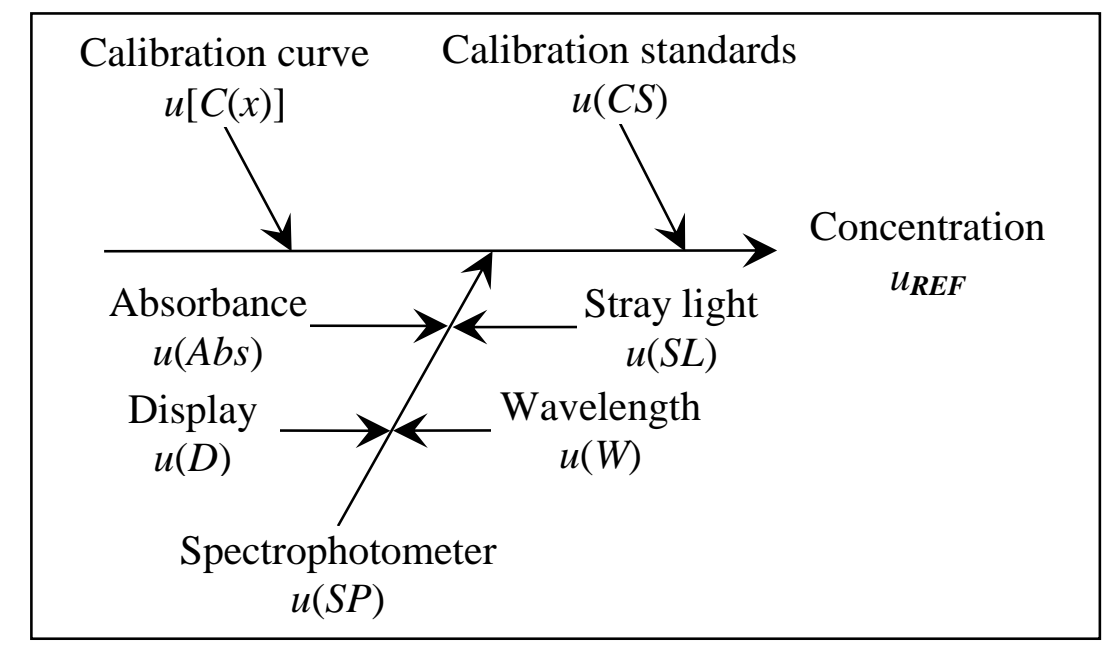

Figure S2. Cause and effect diagram for uncertainty sources of the spectrophotometric method

In (Figure S2) is presented the cause and effect diagram for the uncertainty sources of the spectrophotometric method. 


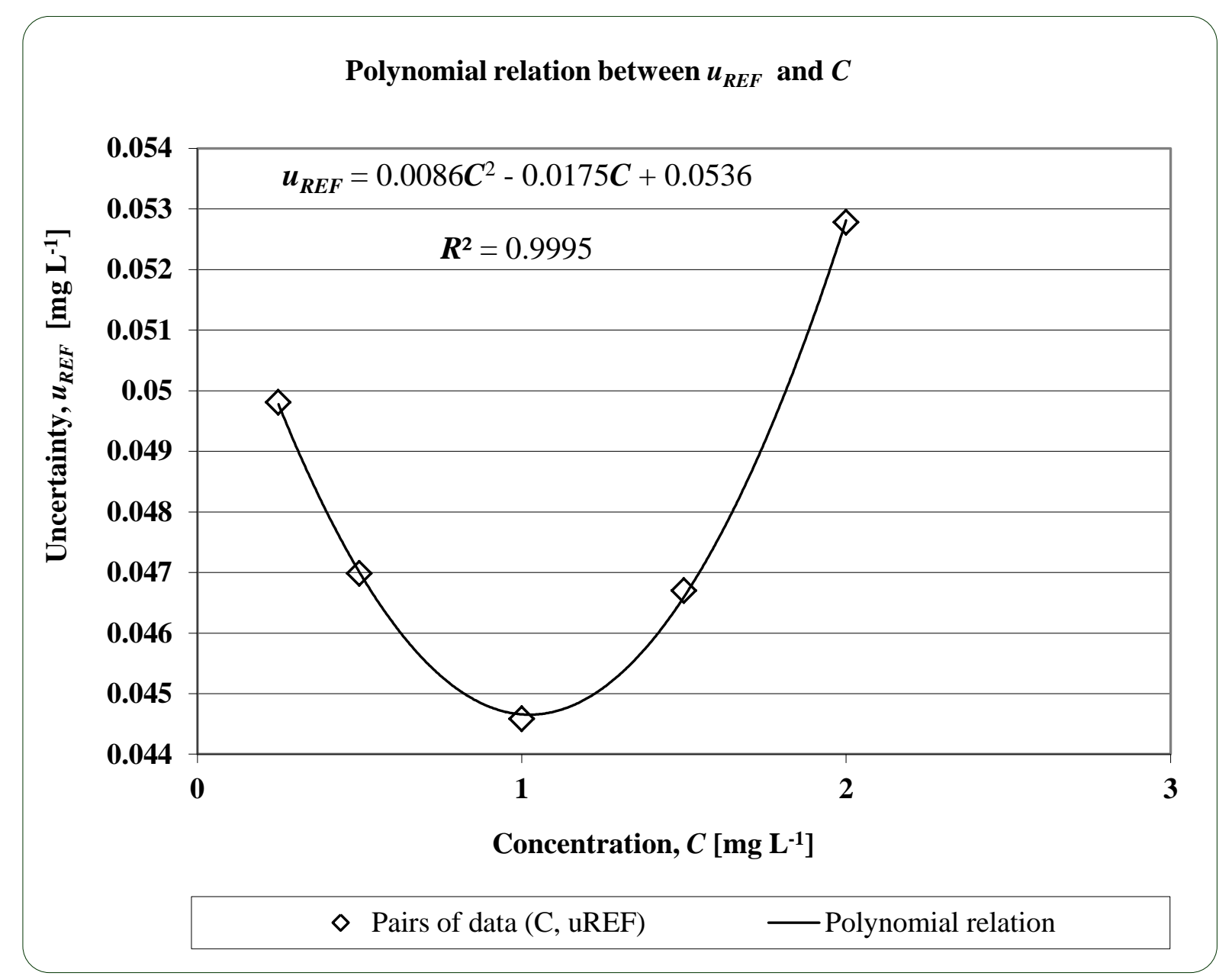

Figure S3. Relation between uncertainty and concentration for the spectrophotometric method

A polynomial relation of grade 2 was detected, plotting the associated uncertainty values versus the concentration values $\left(C\right.$; $\left.u_{\boldsymbol{R} E \boldsymbol{F}}\right)$ for the spectrophotometric method. 